\title{
Chine : génération perdue
}

Michel BonNIN, Génération perdue. Le mouvement d'envoi des jeunes instruits à la campagne en Chine, 1968-1980, Paris, Éditions de l'École des hautes études en sciences sociales, 2004, $491 \mathrm{p}$.

\section{Chloé Froissart}

\section{(2) OpenEdition Journals}

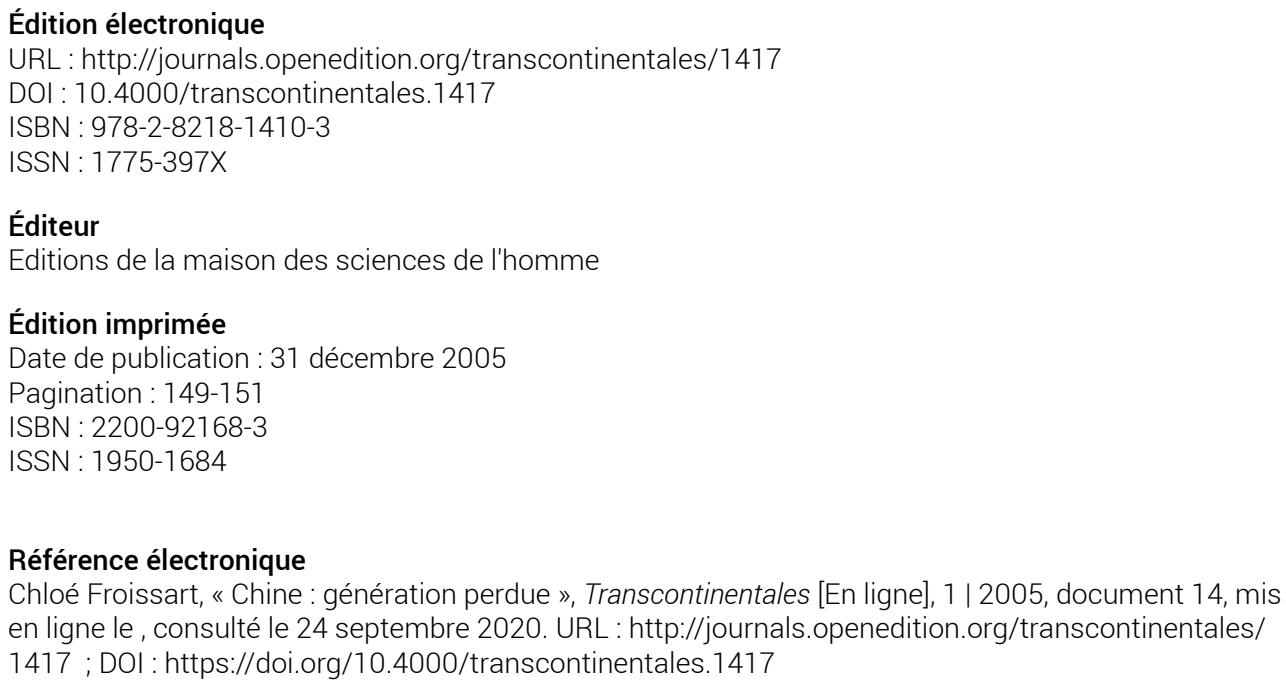
en ligne le, consulté le 24 septembre 2020. URL : http://journals.openedition.org/transcontinentales/ 1417 ; DOI : https://doi.org/10.4000/transcontinentales.1417

Ce document a été généré automatiquement le 24 septembre 2020.

Tous droits réservés 


\section{Chine : génération perdue}

Michel BonNin, Génération perdue. Le mouvement d'envoi des jeunes instruits à la campagne en Chine, 1968-1980, Paris, Éditions de l'École des hautes études en sciences sociales, 2004, $491 \mathrm{p}$.

\section{Chloé Froissart}

\section{RÉFÉRENCE}

Michel BONNIN, Génération perdue. Le mouvement d'envoi des jeunes instruits à la campagne en Chine, 1968-1980, Paris, Éditions de l'École des hautes études en sciences sociales, $2004,491 \mathrm{p}$.

1 Michel Bonnin apporte dans Génération perdue une remarquable contribution à la compréhension du totalitarisme maoïste à travers l'analyse de l'un des mouvements de masse les plus radicaux qu'ait connu le régime: l'envoi à la campagne des jeunes diplômés urbains, à la suite de la Révolution culturelle. Ce mouvement d'envoi à la campagne a concerné 17 millions de jeunes citadins, soit la moitié d'une classe d'âge, appelée à abandonner famille, statut social, études et perspectives de carrière pour se transformer en paysans. Outre son coût humain considérable, ce mouvement a eu de profondes conséquences sur l'évolution économique, politique et idéologique du régime.

2 Les motivations qui ont présidé au lancement et à la poursuite du mouvement étaient avant tout d'ordre politique et idéologique. Il s'agissait pour Mao de mettre un terme à la Révolution culturelle, le mouvement des Gardes rouges échappant de plus en plus au pouvoir, et d'empêcher la formation d'une élite intellectuelle susceptible de remettre en cause le régime. Mao entendait aussi prolonger la révolution et conforter son prestige en lançant un nouveau mouvement. La démonstration va à l'encontre de la thèse de la «rationalité » du mouvement, défendue par certains spécialistes des années 1970, qui y ont vu surtout des motivations économiques (parer au chômage urbain) et démographiques (délester les villes de leur surplus de population). Ces raisons ont bien expliqué le soutien apporté au mouvement par la faction réaliste et 
pragmatiste opposée à Mao, faction conduite par Zhou Enlai et Liu Shaoqi. Michel Bonnin montre toutefois que ces paramètres ne peuvent rendre compte ni de la pérennité du mouvement - étant donné l'importance de son coût économique et de l'exode rural - ni de sa fin, qui a coïncidé avec la mort politique du chef charismatique, au moment le plus défavorable du point de vue démographique et économique. Ce livre offre donc une brillante illustration par le cas chinois de la thèse de Hannah Arendt (Le système totalitaire, 1972), selon laquelle les formations totalitaires cherchent à se maintenir au pouvoir non au moyen d'une gestion rationnelle de la société, mais par sa mise en mouvement perpétuelle.

3 L'auteur parvient à rendre compte de l'ensemble des aspects d'un objet extrêmement complexe en mettant en œuvre une méthode pluridisciplinaire qui fait appel tant à l'histoire politique, culturelle et des mentalités, qu'à l'économie, la démographie et la sociologie. Parmi les sources extrêmement riches et diverses mobilisées par Michel Bonnin, signalons un grand nombre d'entretiens avec des jeunes instruits (zhiqing) qu'il a réalisés à partir du milieu des années 1970 alors que, jeune sinisant à Hong Kong, il s'est trouvé confronté aux premiers réfugiés. L'analyse de ces témoignages, ainsi que celle de la production littéraire des zhiqing, permet de donner une résonance particulièrement vivante à l'expérience de cette génération dans ses dimensions à la fois matérielles et morales, et de l'illustrer par des trajectoires et des tragédies individuelles emblématiques. Cette connaissance intime du vécu des jeunes instruits, et l'important appareil statistique sur lequel s'appuie la démonstration, permettent à l'auteur de confronter le discours officiel, analysé dans la presse et les émissions radiophoniques de l'époque, à la portée réelle du mouvement. L'ouvrage comporte en outre une importante iconographie et un lexique qu'apprécieront les sinisants.

4 Si l'analyse du mouvement contribue à la compréhension de la nature du régime totalitaire chinois, il en révèle aussi les limites. L'auteur montre que le mouvement a été mis à mal par la résistance de la société et n'a pas permis la formation de la relève révolutionnaire que Mao appelait de ses vœux. Il a au contraire donné naissance à une génération profondément anti-maoïste et désabusée. Enfin, les conséquences socioéconomiques du mouvement ont favorisé la prise de pouvoir par la faction pragmatiste conduite par Deng Xiaoping, et le lancement des réformes cherchant à sortir le pays de la crise. Si le bilan du mouvement à court terme dépeint une génération sacrifiée aux luttes de pouvoir au sommet et révèle l'inutilité de ce sacrifice au regard des raisons invoquées pour le justifier, le bilan historique à long terme montre au contraire qu'il a joué un rôle essentiel dans la sortie de la Chine du maoïsme.

5 Le livre se ferme sur une discussion de la notion de génération. On aurait aimé qu'il ouvre plus franchement sur le devenir de cette génération dans la Chine contemporaine. En effet, comme l'auteur l'évoque brièvement, une partie des zhiqing parvenus à reprendre leurs études ont eu un rôle décisif au cours des années 1980 dans l'évolution politique, idéologique et culturelle de la Chine, certains prenant directement part au processus de réformes. Dans les années 1990, ces zhiqing ont donné naissance à un courant de pensée connu en Chine sous le nom de libéralisme politique. Aujourd'hui encore, bon nombre de chercheurs, d'intellectuels engagés et de travailleurs sociaux font partie de cette génération et continuent de mettre en pratique les idéaux qui étaient les siens : servir le peuple, participer au développement du pays et s'unir aux masses. Cet engagement intellectuel et social qui perdure de nos jours est sans doute l'un des seuls moyens qui reste à cette génération, dont la parole est 
politiquement bannie et socialement taboue, de transmettre son expérience et de briser son isolement. Mais à côté de ceux qui ont réussi à donner sens à leur sacrifice, il y a ceux qui furent définitivement perdus, comme ces jeunes instruits rentrés en ville grâce à l'obtention d'un emploi d'ouvrier dans le secteur public, et qui ont été les premiers à faire les frais des réformes économiques.

6 Les non-spécialistes devraient trouver profit à cet ouvrage, certes dense et foisonnant, en raison de la structure extrêmement claire et didactique de l'ensemble. Quant aux sinologues, il leur est donné là un outil de travail indispensable.

\section{AUTEURS}

\section{CHLOÉ FROISSART}

Centre d'études français sur la Chine contemporaine, Hong Kong 